\title{
TAIL ASYMPTOTICS FOR THE QUEUE SIZE DISTRIBUTION IN AN M/G/1 RETRIAL QUEUE
}

\author{
JERIM KIM ${ }^{* * *}$ AND \\ BARA KIM, ${ }^{* * *}$ Korea University \\ SUNG-SEOK KO, ${ }^{* * * *}$ Konkuk University
}

\begin{abstract}
We consider an M/G/1 retrial queue, where the service time distribution has a finite exponential moment. We show that the tail of the queue size distribution is asymptotically given by a geometric function multiplied by a power function. The result is obtained by investigating analytic properties of probability generating functions for the queue size and the server state.
\end{abstract}

Keywords: M/G/1 retrial queue; tail asymptotics; queue size distribution

2000 Mathematics Subject Classification: Primary 60K25

\section{Introduction}

Retrial phenomena arise in many practical situations such as in call center systems and many other telecommunication systems. Retrial queues, which deal with stochastic models for the retrial phenomena, have been investigated for several decades. Detailed overviews for retrial queues can be found in [3], [4], and [8].

In this paper we consider an M/G/1 retrial queueing system, where customers arrive according to a Poisson process with intensity $\lambda$ and the service times $B$ for customers are independent and identically distributed with Laplace-Stieltjes transform $\beta$, i.e. $\beta(s):=\mathrm{E}\left[\mathrm{e}^{-s B}\right]$. If the server is idle at the time of a customer arrival, the arriving customer begins to be served immediately and leaves the system after service completion. Otherwise, i.e. if the server is busy, the arriving customer joins a retrial group, called an orbit. While in orbit, each customer spends an exponential time with mean $v^{-1}$ before visiting the server again. If an incoming repeated customer from the orbit finds the server idle, it is served and leaves the system after service completion. Otherwise, i.e. if the repeated customer finds the server busy, the customer comes back to the orbit immediately and tries again after an exponential time with mean $v^{-1}$. The traffic load $\rho$ is defined as $\rho=\lambda \mathrm{E} B$. It is assumed that $\rho<1$ for stability of the system.

The interest of this paper is the light-tailed asymptotics of the queue size distribution in the $\mathrm{M} / \mathrm{G} / 1$ retrial queue, when the service time distribution has a finite exponential moment, i.e.

$$
\gamma:=\sup \left\{t \in \mathbb{R}: \mathrm{Ee}^{t B}<\infty\right\}>0 .
$$

For the usual M/G/1 queue, it is well known that the stationary distribution of the queue size has a geometric tail if (1) holds with a related additional assumption. More precisely, for the

Received 3 July 2007; revision received 23 September 2007.

* Postal address: Department of Mathematics, Korea University, 1 Anam-dong, Sungbuk-ku, Seoul 136-701, Korea.

** Email address: b1155@ hanmail.net

*** Email address: bara@korea.ac.kr

**** Postal address: Department of Industrial Engineering, Konkuk University, 1 Hwayang-dong, Gwangjin-Gu, Seoul 143-701, Korea. Email address: ssko@konkuk.ac.kr 
usual M/G/1 queue with arrival rate $\lambda$ and service times $B$, if there is a real number $\sigma$ satisfying

$$
\beta(\lambda-\lambda \sigma)=\sigma, \quad 1<\sigma<1+\frac{\gamma}{\lambda},
$$

then the queue size $Q$ at steady state satisfies

$$
\mathrm{P}(Q=n) \sim c \sigma^{-n} \quad \text { as } n \rightarrow \infty
$$

with a constant $c>0$. Here and subsequently, $g_{n} \sim h_{n}$ denotes $\lim _{n \rightarrow \infty}\left(g_{n} / h_{n}\right)=1$. See, for example, [1], [2], [10], [11], [13], [15], [17], and the references therein for geometric tails of the queue size distribution in the usual M/G/1 or further G/G/1 queue. However, for the retrial queue, it does not seem that there is any known result on light-tailed asymptotics of the queue size distribution in the open literature. The situation is not much different for the light-tailed asymptotics of the waiting time distribution in retrial queues. The only accessible related work seems to be [12], where a light-tailed approximation was suggested for the waiting time distribution in the $\mathrm{M} / \mathrm{G} / 1$ retrial queue.

On the other hand, there are recent works on heavy-tailed asymptotics in the M/G/1 retrial queue: Shang et al. [14] showed that the stationary distribution of the queue length in the $\mathrm{M} / \mathrm{G} / 1$ retrial queue is subexponential if the stationary distribution of the queue length in the corresponding ordinary M/G/1 queue is subexponential. As a corollary of this property, Shang et al. proved that the stationary distribution of the queue length in the M/G/1 retrial queue has a regularly varying tail if the service time distribution has a regularly varying tail. Kim and Kim [9] showed that if the service time distribution has a regularly varying tail of index $-\alpha, 1<\alpha<2$, in the M/G/1 retrial queue, then the waiting time distribution has a regularly varying tail of index $1-\alpha$.

The main contribution of this paper is to find the light-tailed asymptotics for the queue size distribution in the M/G/1 retrial queue. More precisely, we show that if (2) holds then

$$
\begin{gathered}
\mathrm{P}(C=0, N=n) \sim c_{0} n^{a-1} \sigma^{-n} \quad \text { as } n \rightarrow \infty, \\
\mathrm{P}(C=1, N=n) \sim c_{1} n^{a} \sigma^{-n} \quad \text { as } n \rightarrow \infty,
\end{gathered}
$$

for positive constants $a, c_{0}$, and $c_{1}$, where $N$ is the number of customers in the orbit at steady state and $C$ is 0 if the server is idle and 1 otherwise. Furthermore, the constants $a, c_{0}$, and $c_{1}$ are given explicitly. The results (3) and (4) are obtained by investigating probability generating functions (PGFs) for $C$ and $N$ through decomposing a component of the PGFs as a sum of the principal part and the analytic part. The method of obtaining asymptotic expansions of state probabilities from generating functions using complex function theory can be found in Tijms [16]. Tijms illustrated the method in many queueing models. Further illustrations are found in [5] and [6], for example.

The remainder of this paper is organized as follows. In Section 2 we study the PGFs for $C$ and $N$. Specifically, we decompose a component of the PGFs as a sum of principal part and the analytic part. The decomposition plays a crucial role in deriving the light-tailed asymptotics for the queue size distribution in Section 3. The main results (3) and (4) are provided with explicit expressions for $c_{0}$ and $c_{1}$ in Section 3. The results (3) and (4) are examined for the $\mathrm{M} / \mathrm{M} / 1$ retrial queue. 


\section{Probability generating function of the queue size}

Consider the M/G/1 retrial queue at steady state. We suppose that (2) holds. Recall that

$$
\begin{gathered}
C= \begin{cases}0 & \text { if the server is idle, } \\
1 & \text { if the server is busy, }\end{cases} \\
N=\text { the number of customers in the orbit. }
\end{gathered}
$$

We define generating functions $P_{0}$ and $P_{1}$ as follows:

$$
\begin{aligned}
& P_{0}(z):=\mathrm{E}\left(z^{N} ; C=0\right)=\sum_{n=0}^{\infty} \mathrm{P}(N=n, C=0) z^{n}, \\
& P_{1}(z):=\mathrm{E}\left(z^{N} ; C=1\right)=\sum_{n=0}^{\infty} \mathrm{P}(N=n, C=1) z^{n} .
\end{aligned}
$$

It is well known (see, for example, [7] or [8, Theorem 1.2]) that the generating functions $P_{0}$ and $P_{1}$ are given by

$$
\begin{gathered}
P_{0}(z)=(1-\rho) \exp \left(\frac{\lambda}{v} \int_{1}^{z} \frac{1-\beta(\lambda-\lambda u)}{\beta(\lambda-\lambda u)-u} \mathrm{~d} u\right), \quad|z|<\sigma, \\
P_{1}(z)=(1-\rho) \frac{1-\beta(\lambda-\lambda z)}{\beta(\lambda-\lambda z)-z} \exp \left(\frac{\lambda}{v} \int_{1}^{z} \frac{1-\beta(\lambda-\lambda u)}{\beta(\lambda-\lambda u)-u} \mathrm{~d} u\right), \quad|z|<\sigma,
\end{gathered}
$$

where, for $z=1,(1-\beta(\lambda-\lambda z)) /(\beta(\lambda-\lambda z)-z)$ is interpreted as

$$
\lim _{z \rightarrow 1} \frac{1-\beta(\lambda-\lambda z)}{\beta(\lambda-\lambda z)-z}=\frac{\rho}{1-\rho} .
$$

We remark that an interesting probabilistic derivation of (5) and (6) was provided in [7].

To investigate the light-tailed behavior of the queue size distribution, we need to have a close look at singularities of $(1-\beta(\lambda-\lambda z)) /(\beta(\lambda-\lambda z)-z)$ on $\{z \in \mathbb{C}:|z|<1+\gamma / \lambda\}$. Lemma 1 , below, locates the 0 s of $\beta(\lambda-\lambda z)-z,|z| \leq \sigma$.

Lemma 1. The analytic function $\beta(\lambda-\lambda z)-z,|z|<1+\gamma / \lambda$, has simple zeros at 1 and $\sigma$. Furthermore, it has no other 0 s on $\{z \in \mathbb{C}:|z| \leq \sigma\}$.

Proof. Let $f(z)=\beta(\lambda-\lambda z)-z,|z|<1+\gamma / \lambda$. We observe that 1 is a zero of $f$. Furthermore, the zero 1 is simple because $f^{\prime}(1)=\rho-1<0$. Since $f(z)$ is strictly convex in $z$ on $(0,1+\gamma / \lambda)$ and $f(1)=f(\sigma)=0$, we have $f(z)<0$ for $1<z<\sigma$. Thus,

$$
\beta(\lambda-\lambda z)<z, \quad 1<z<\sigma .
$$

Therefore, for $\eta \in(1, \sigma)$,

$$
|f(z)+z|=|\beta(\lambda-\lambda z)|<\beta(\lambda-\lambda|z|)<|z| \quad \text { if }|z|=\eta .
$$

Hence, Rouché's theorem asserts that $f(z)$ has no 0 s on $\{z \in \mathbb{C}:|z| \leq \eta\}$ except 1 , which is a simple zero. Letting $\eta \rightarrow \sigma-$ asserts that $f(z)$ has no 0 s on $\{z \in \mathbb{C}:|z|<\sigma\}$ except the simple 
zero 1 . We know that $\sigma$ is a zero of $\beta(\lambda+\lambda z)-z$. Since $f(z)$ is convex in $z$ on $(0,1+\gamma / \lambda)$ and $f(1)=f(\sigma)=0$, we have $f^{\prime}(\sigma)>0$. Thus, the zero $\sigma$ of $f$ is simple. Finally,

$$
|\beta(\lambda-\lambda z)|<\beta(\lambda-\lambda|z|)=|z| \quad \text { if }|z|=\sigma \text { and } z \neq \sigma .
$$

Hence, $f$ has no 0 s on $\{z \in \mathbb{C}:|z|=\sigma, z \neq \sigma\}$, which completes the proof.

Lemma 2 , below, decomposes $(1-\beta(\lambda-\lambda z)) /(\beta(\lambda-\lambda z)-z)$ as a sum of the principal part at $z=\sigma$ and the analytic part.

Lemma 2. There exists $\delta \in(0,1+\gamma / \lambda-\sigma)$ and an analytic function $\Xi$ on $\{z \in \mathbb{C}:|z|<\sigma+\delta\}$ such that

$$
\frac{1-\beta(\lambda-\lambda z)}{\beta(\lambda-\lambda z)-z}=-\frac{\sigma-1}{-\lambda \beta^{\prime}(\lambda-\lambda \sigma)-1} \frac{1}{z-\sigma}+\Xi(z), \quad|z|<\sigma+\delta, \quad z \neq \sigma .
$$

Proof. We note that $(1-\beta(\lambda-\lambda z)) /(\beta(\lambda-\lambda z)-z)$ has a removable singularity at $z=1$ and is regarded as analytic at $z=1$. By Lemma 1 , there exists $\delta>0$ such that $(1-\beta(\lambda-\lambda z)) /$ $(\beta(\lambda-\lambda z)-z)$ is analytic on $\{z \in \mathbb{C}:|z|<\sigma+\delta, z \neq \sigma\}$. We observe that $(1-\beta(\lambda-\lambda z)) /$ $(\beta(\lambda-\lambda z)-z)$ has a simple pole at $z=\sigma$ and the residue of $(1-\beta(\lambda-\lambda z)) /(\beta(\lambda-\lambda z)-z)$ at $z=\sigma$ is $-(\sigma-1) /\left(-\lambda \beta^{\prime}(\lambda-\lambda \sigma)-1\right)$. Therefore,

$$
\frac{1-\beta(\lambda-\lambda z)}{\beta(\lambda-\lambda z)-z}+\frac{\sigma-1}{-\lambda \beta^{\prime}(\lambda-\lambda \sigma)-1} \frac{1}{z-\sigma}
$$

has a removable singularity at $z=\sigma$. Letting

$$
\Xi(z)= \begin{cases}\frac{1-\beta(\lambda-\lambda z)}{\beta(\lambda-\lambda z)-z}+\frac{\sigma-1}{-\lambda \beta^{\prime}(\lambda-\lambda \sigma)-1} \frac{1}{z-\sigma}, & |z|<\sigma+\delta, z \neq \sigma, \\ \lim _{u \rightarrow \sigma} \frac{1-\beta(\lambda-\lambda u)}{\beta(\lambda-\lambda u)-u}+\frac{\sigma-1}{-\lambda \beta^{\prime}(\lambda-\lambda \sigma)-1} \frac{1}{u-\sigma}, & z=\sigma,\end{cases}
$$

completes the proof.

\section{Tail asymptotics for the queue size distribution}

In this section we state and prove the main result of this paper which provides the tail asymptotics of the queue size distribution in the M/G/1 retrial queue when condition (2) holds.

Theorem 1. If (2) holds then

$$
\begin{gathered}
\mathrm{P}(C=0, N=n) \sim c_{0} n^{a-1} \sigma^{-n} \quad \text { as } n \rightarrow \infty, \\
\mathrm{P}(C=1, N=n) \sim c_{1} n^{a} \sigma^{-n} \text { as } n \rightarrow \infty,
\end{gathered}
$$

where

$$
\begin{aligned}
a & =\frac{\lambda}{v} \frac{\sigma-1}{-\lambda \beta^{\prime}(\lambda-\lambda \sigma)-1}, \\
c_{0} & =\frac{1-\rho}{\Gamma(a)}\left(\frac{\sigma-1}{\sigma}\right)^{a} \exp \left(\int_{1}^{\sigma} \frac{\lambda}{v} \frac{1-\beta(\lambda-\lambda z)}{\beta(\lambda-\lambda z)-z}+\frac{a}{z-\sigma} \mathrm{d} z\right), \\
c_{1} & =\frac{\nu c_{0}}{\lambda \sigma},
\end{aligned}
$$

and $\Gamma(\cdot)$ denotes the gamma function. 
Proof. First we prove (8). Substituting (7) into (5) yields

$$
P_{0}(z)=(1-\rho)\left(\frac{\sigma-z}{\sigma-1}\right)^{-a} \exp \left(\frac{\lambda}{v} \int_{1}^{z} \Xi(u) \mathrm{d} u\right), \quad|z|<\sigma .
$$

Since $\Xi(z)$ is analytic on $\{z \in \mathbb{C}:|z|<\sigma+\delta\}$, so is $\exp \left((\lambda / \nu) \int_{1}^{z} \Xi(u) \mathrm{d} u\right)$. Let $\sum_{n=0}^{\infty} \phi_{n} z^{n}$ be the power series expansion of $\exp \left((\lambda / \nu) \int_{1}^{z} \Xi(u) \mathrm{d} u\right)$ at $z=0$, i.e.

$$
\exp \left(\frac{\lambda}{v} \int_{1}^{z} \Xi(u) \mathrm{d} u\right)=\sum_{n=0}^{\infty} \phi_{n} z^{n}, \quad|z|<\sigma+\delta
$$

We note that $(1-\rho)((\sigma-z) /(\sigma-1))^{-a}$ has the following power series expansion at $z=0$ :

$$
(1-\rho)\left(\frac{\sigma-z}{\sigma-1}\right)^{-a}=(1-\rho)\left(\frac{\sigma-1}{\sigma}\right)^{a} \sum_{n=0}^{\infty} \frac{\Gamma(a+n)}{\Gamma(a) \Gamma(n+1)} \sigma^{-n} z^{n}, \quad|z|<\sigma .
$$

Substituting (11) and (12) into (10) yields

$$
\mathrm{P}(C=0, N=n)=(1-\rho)\left(\frac{\sigma-1}{\sigma}\right)^{a} \sum_{k=0}^{n} \frac{\Gamma(a+n-k)}{\Gamma(a) \Gamma(n-k+1)} \sigma^{k-n} \phi_{k}, \quad n=0,1,2, \ldots
$$

Therefore,

$$
\begin{aligned}
& \frac{\mathrm{P}(C=0, N=n)}{(\Gamma(a+n) / \Gamma(a) \Gamma(n+1)) \sigma^{-n}} \\
& =(1-\rho)\left(\frac{\sigma-1}{\sigma}\right)^{a} \sum_{k=0}^{n} \frac{\Gamma(a+n-k) \Gamma(n+1)}{\Gamma(a+n) \Gamma(n-k+1)} \phi_{k} \sigma^{k} \\
& =(1-\rho)\left(\frac{\sigma-1}{\sigma}\right)^{a} \sum_{k=0}^{\infty} \mathbf{1}_{\{k \leq n\}} \frac{n(n-1) \cdots(n-k+1)}{(a+n-1)(a+n-2) \cdots(a+n-k)} \phi_{k} \sigma^{k}
\end{aligned}
$$

where

$$
\mathbf{1}_{\{k \leq n\}}= \begin{cases}1 & \text { if } k \leq n \\ 0 & \text { otherwise }\end{cases}
$$

If $a \geq 1$ then

$$
\mathbf{1}_{\{k \leq n\}} \frac{n(n-1) \cdots(n-k+1)}{(a+n-1)(a+n-2) \cdots(a+n-k)}<1 .
$$

If $0<a<1$ then

$$
\begin{aligned}
& \mathbf{1}_{\{k \leq n\}} \frac{n(n-1) \cdots(n-k+1)}{(a+n-1)(a+n-2) \cdots(a+n-k)}=\mathbf{1}_{\{k \leq n\}} \frac{n}{a+n-1} \frac{n-1}{a+n-2} \cdots \frac{n-k+1}{a+n-k} \\
& \leq \frac{k}{a+k-1} \frac{k-1}{a+k-2} \cdots \frac{1}{a} \\
& \leq \frac{k}{a}
\end{aligned}
$$


Hence, for all $n \geq 0$ and $k \geq 0$,

$$
\left|\mathbf{1}_{\{k \leq n\}} \frac{n(n-1) \cdots(n-k+1)}{(a+n-1)(a+n-2) \cdots(a+n-k)} \phi_{k} \sigma^{k}\right| \leq\left(\frac{1}{a} k+1\right)\left|\phi_{k}\right| \sigma^{k} .
$$

Since $\exp \left((\lambda / \nu) \int_{1}^{z} \Xi(u) \mathrm{d} u\right)$ is analytic on $\{z \in \mathbb{C}:|z|<\sigma+\delta\}$, the radius of convergence of the power series $\sum_{n=0}^{\infty} \phi_{n} z^{n}$ is not less then $\sigma+\delta$. Therefore,

$$
\sum_{k=0}^{\infty}\left(\frac{1}{a} k+1\right)\left|\phi_{k}\right| \sigma^{k}<\infty \text {. }
$$

By (15) and (16), letting $n$ tend to $\infty$ in (14) with the Lebesgue dominated convergence theorem yields

$$
\begin{aligned}
\lim _{n \rightarrow \infty} \frac{\mathrm{P}(C=0, N=n)}{(\Gamma(a+n) / \Gamma(a) \Gamma(n+1)) \sigma^{-n}} & =(1-\rho)\left(\frac{\sigma-1}{\sigma}\right)^{a} \sum_{k=0}^{\infty} \phi_{k} \sigma^{k} \\
& =(1-\rho)\left(\frac{\sigma-1}{\sigma}\right)^{a} \exp \left(\frac{\lambda}{v} \int_{1}^{\sigma} \Xi(u) \mathrm{d} u\right) \\
& =\Gamma(a) c_{0} .
\end{aligned}
$$

Since $\Gamma(a+n) / \Gamma(n+1) \sim n^{a-1}$ as $n \rightarrow \infty,(17)$ is equivalent to (8).

Next we prove (9). Substituting (7) into (6) yields

$$
\begin{aligned}
P_{1}(z)= & \frac{1-\rho}{-\sigma \beta^{\prime}(\lambda-\lambda \sigma)-1}\left(\frac{\sigma-z}{\sigma-1}\right)^{-a-1} \exp \left(\frac{\lambda}{v} \int_{1}^{z} \Xi(u) \mathrm{d} u\right) \\
& +(1-\rho)\left(\frac{\sigma-z}{\sigma-1}\right)^{-a} \Xi(z) \exp \left(\frac{\lambda}{v} \int_{1}^{z} \Xi(u) \mathrm{d} u\right), \quad|z|<\sigma .
\end{aligned}
$$

Now we consider the power series expansions of the two terms on the right-hand side of (18) separately as follows:

$$
\begin{gathered}
\frac{1-\rho}{-\lambda \beta^{\prime}(\lambda-\lambda \sigma)-1}\left(\frac{\sigma-z}{\sigma-1}\right)^{-a-1} \exp \left(\frac{\lambda}{v} \int_{1}^{z} \Xi(u) \mathrm{d} u\right)=\sum_{k=0}^{\infty} p_{n} z^{n}, \\
(1-\rho)\left(\frac{\sigma-z}{\sigma-1}\right)^{-a} \Xi(z) \exp \left(\frac{\lambda}{v} \int_{1}^{z} \Xi(u) \mathrm{d} u\right)=\sum_{k=0}^{\infty} q_{n} z^{n}
\end{gathered}
$$

By the same method which we used to derive (8), we obtain

$$
\begin{gathered}
p_{n} \sim c_{1} n^{a} \sigma^{-n}, \\
q_{n} \sim c_{0} \Xi(\sigma) n^{a-1} \sigma^{-n} .
\end{gathered}
$$

Therefore, $q_{n}=o\left(p_{n}\right)$ as $n$ tends to $\infty$, and

$$
\mathrm{P}(C=1, N=n)=p_{n}+q_{n} \sim c_{1} n^{a} \sigma^{-n} \text { as } n \rightarrow \infty .
$$


By Theorem 1 we have

$$
\begin{aligned}
\mathrm{P}(N=n) & \sim \mathrm{P}(C=1, N=n) \quad \text { as } n \rightarrow \infty, \\
\mathrm{P}(C+N=n) & \sim \mathrm{P}(C=1, N=n-1) \quad \text { as } n \rightarrow \infty .
\end{aligned}
$$

Therefore, the following corollary is immediate.

Corollary 1. If (2) holds then the queue size, $N$, and the number of customers in the system, $C+N$, have distributions with the following tail asymptotics:

$$
\begin{aligned}
\mathrm{P}(N=n) & \sim c_{1} n^{a} \sigma^{-n} \quad \text { as } n \rightarrow \infty, \\
\mathrm{P}(C+N=n) & \sim c_{1} n^{a} \sigma^{-n+1} \quad \text { as } n \rightarrow \infty .
\end{aligned}
$$

Remark. Suppose that the service times $B$ have exponential distribution with mean $\mu^{-1}$. Then we have

$$
\sigma=\frac{1}{\rho}, \quad a=\frac{\lambda}{\nu}, \quad c_{0}=\frac{(1-\rho)^{\lambda / \nu+1}}{\Gamma(\lambda / \nu)}, \quad \text { and } \quad c_{1}=\frac{\rho(1-\rho)^{\lambda / \nu+1}}{\Gamma(\lambda / \nu+1)} .
$$

Hence, Theorem 1 asserts that

$$
\begin{aligned}
& \mathrm{P}(C=0, N=n) \sim \frac{(1-\rho)^{\lambda / \nu+1}}{\Gamma(\lambda / \nu)} n^{\lambda / \nu-1} \rho^{n} \quad \text { as } n \rightarrow \infty, \\
& \mathrm{P}(C=1, N=n) \sim \frac{\rho(1-\rho)^{\lambda / \nu+1}}{\Gamma(\lambda / \nu+1)} n^{\lambda / \nu} \rho^{n} \quad \text { as } n \rightarrow \infty .
\end{aligned}
$$

The results (19) and (20) are consistent with the following known result (see [8, Theorem 1.1]):

$$
\begin{aligned}
& \mathrm{P}(C=0, N=n)=\frac{(1-\rho)^{\lambda / v+1}}{v n !} \prod_{k=0}^{n-1}\left(\frac{\lambda}{v}+k\right) \rho^{n}, \quad n=0,1,2, \ldots, \\
& \mathrm{P}(C=1, N=n)=\frac{\rho(1-\rho)^{\lambda / v+1}}{n !} \prod_{k=1}^{n}\left(\frac{\lambda}{v}+k\right) \rho^{n}, \quad n=0,1,2, \ldots
\end{aligned}
$$

\section{Acknowledgement}

This research was supported by the Ministry of Information and Communication, Korea, under the Information Technology Research Center support program supervised by the Institute of Information Technology Assessment.

\section{References}

[1] Abate, J. And Whitt, W. (1997). Asymptotics for M/G/1 low-priority waiting-time tail probabilities. Queueing Systems 26, 69-104.

[2] Abate, J., Choudhury, G. L. And Whitt, W. (1995). Exponential approximations for tail probabilities in queues. I. Waiting times. Operat. Res. 43, 885-901.

[3] Artalejo, J. R. (1999). A classified bibliography of research on retrial queues: progress in 1990-1999. TOP 7, 187-211.

[4] Choi, B. D. And Chang, Y. (1999). Single server retrial queues with priority calls. Math. Comput. Modelling 30, 7-32.

[5] CHOI, B. D. AND KIM, B. (2000). Sharp result on convergence rate for the distribution of GI/M/1/K queues as $K$ tends to infinity. J. Appl. Prob. 37, 1010-1019. 
[6] Chol, B. D., KIm, B. And Wee, I.-S. (2000). Asymptotic behavior of loss probability in GI/M/1/K queue as $K$ tends to infinity. Queueing Systems 36, 437-442.

[7] De KoK, A. G. (1984). Algorithmic methods for single server systems with repeated attempts. Statistica Neerlandica 38, 23-32.

[8] Falin, G. I. and Templeton, J. G. C. (1997). Retrial Queues. Chapman \& Hall, London.

[9] KIm, J. AND KIM, B. (2007). Regularly varying tail of the waiting time distribution in M/G/1 retrial queue. Submitted.

[10] LI, Q.-L. AND ZHAO, Y. Q. (2005). Light-tailed asymptotics of stationary probability vectors of Markov chains of GI/G/1 type. Adv. Appl. Prob. 37, 1075-1093.

[11] Miyazawa, M. And ZHao, Y. Q. (2004). The stationary tail asymptotics in the GI/G/1-type queue with countably many background states. Adv. Appl. Prob. 36, 1231-1251.

[12] Nobel, R. D. and Tisms, H. C. (2006). Waiting-time probabilities in the M/G/1 retrial queue. Statistica Neerlandica 60, 73-78.

[13] Sakurai, T. (2004). Approximating M/G/1 waiting time tail probabilities. Stoch. Models 20, 173-191.

[14] Shang, W., LiU, L. AND LI, Q. (2006). Tail asymptotics for the queue length in an M/G/1 retrial queue. Queueing Systems 52, 193-198.

[15] Takine, T. (2004). Geometric and subexponential asymptotics of Markov chains of M/G/1 type. Math. Operat. Res. 29, 624-648.

[16] Tisms, H. C. (1987). Stochastic Modelling and Analysis: A Computational Approach. John Wiley, Chichester.

[17] Willmot, G. (1988). A note on the equilibrium M/G/1 queue length. J. Appl. Prob. 25, 228-231. 